\title{
Process Research and Scale-up of the K-Opioid Receptor Agonist CJ-15,161 Drug Candidate
}

\author{
Brian M. Andresen, Stéphane Caron, Michel Couturier*, Keith M. DeVries, Nga M. Do, Kristina \\ Dupont-Gaudet, Arun Ghosh*, Melina Girardin, Joel M. Hawkins, Teresa M. Makowski, Maxime \\ Riou, Janice E. Sieser, John L. Tucker, Brian C. Vanderplas, and Timothy J.N. Watson
}

\begin{abstract}
This account depicts strategies adopted during the development of the $\kappa$-opioid receptor agonist CJ15,161 . While the original discovery synthesis was enabled for scale-up, concomitant process research aimed at identifying a novel and more efficient route was undertaken. In the former case, an efficient four-step sequence has been developed, where the process features four consecutive regioselective and stereospecific inversions at a single aziridinium stereogenic center, which leads to overall retention of stereochemistry in a single operation. The search for novel routes has also resulted in two converging methods involving efficient intermolecular $\mathrm{N}$-arylation strategies. The first approach involves $\mathrm{Pd}$-catalyzed intermolecular $\mathrm{N}$-arylation of an appropriately functionalized diamine, obtained from the precursor $\alpha$-amino acids or, more conveniently, from the corresponding 1,2-amino alcohols. The second approach exploits efficient intermolecular $\mathrm{N}$-arylation of oxazolidinones using catalytic copper in the presence of a bidentate ligand leading to a straightforward and practical synthesis of CJ-15,161.
\end{abstract}

Keywords: N-Arylation · Aziridinium · Copper · Oxazolidinone · Palladium · Process chemistry

\section{Introduction}

The syntheses of drug candidates originally discovered by medicinal chemists are most often not amenable to larger scale preparation. Although the ultimate goal is to develop the optimal manufacturing process, the requisite research activities are time consuming and labor intensive, and therefore not appropriate for the preparation of bulk material required for early studies. For obvious reasons, time is of essence to progress the drug candidate rapidly through the development echelons. With less than perfect chemistry in hand, one must balance re-

${ }^{\star}$ Correspondence: Dr. M. Couturier, Dr. A. Ghosh Pfizer, Inc.

Chemical Research and Development

Eastern Point Road

Groton, CT 06340, USA

Tel.: +1 8604411735 (Couturier)

Fax: +1 860 441-3788

E-Mail: michel.a.couturier@pfizer.com

Tel.: +1 8607156080 (Ghosh)

Fax: +1 860 441-3788

E-Mail: arun.ghosh@pfizer.com sources in such a way as not to impede rapid bulk manufacture to support ongoing development activities, all the while developing a robust manufacturing process should the drug candidate make it to the market. Such was the case in the development program of CJ-15,161 (1), a selective $\kappa$-opioid receptor agonist drug candidate as a potentially nonaddictive analgesic [1].

At the outset, the original discovery route was deemed straightforward from a bond disconnection point of view, but was not amenable to large-scale preparation and long-term manufacture (Scheme 1). More specifically, there were no crystalline intermediates throughout the synthesis, the styrene oxide ring opening by the pyrrolidine lacked regioselectivity, the reactions did not lend themselves to easy manipulations, and the actual drug substance was an amorphous hydrochloride salt. Herein, we describe process improvements made on this original synthesis and strategies adopted to enable timely bulk production for supporting toxicological screening and early clinical trials, together with concomitant process research activities aimed at developing a fundamentally different synthetic strategy that would present synthetic advantages as well as ensure our freedom to operate in this area.

\subsection{Enabling the Original Discovery} Route for Scale-up [2]

As mentioned above, the major drawback of the original discovery synthesis was the total lack of isolable crystalline intermediates requiring several chromatographic separations. Hence, one of our first goals was to identify a crystalline final form for the drug substance, and salt screens showed that the benzoic acid salt mono-hydrate was a stable polymorph suitable for formulation. We therefore had a non-chromatographic means to purify the drug substance. With this mindset, we then sought an alternate protecting group that would impart crystallinity to the penultimate intermediate and would allow purification by simple crystallization, as this was not the case with the original MOM-protected intermediate 4. A crystalline penultimate intermediate would offer an additional safety net in terms of reaching desired specifications for the drug substance as impurity removal could be achieved prior to the last step. The additional driver in changing the MOM protecting group was to remove sodium hydride and chloromethyl methyl ether, a known carcinogen, from the process. Due to time constraints, we deemed that derivatization of pyrrolidinol 3 with several protecting groups, and advancing these to the penul- 


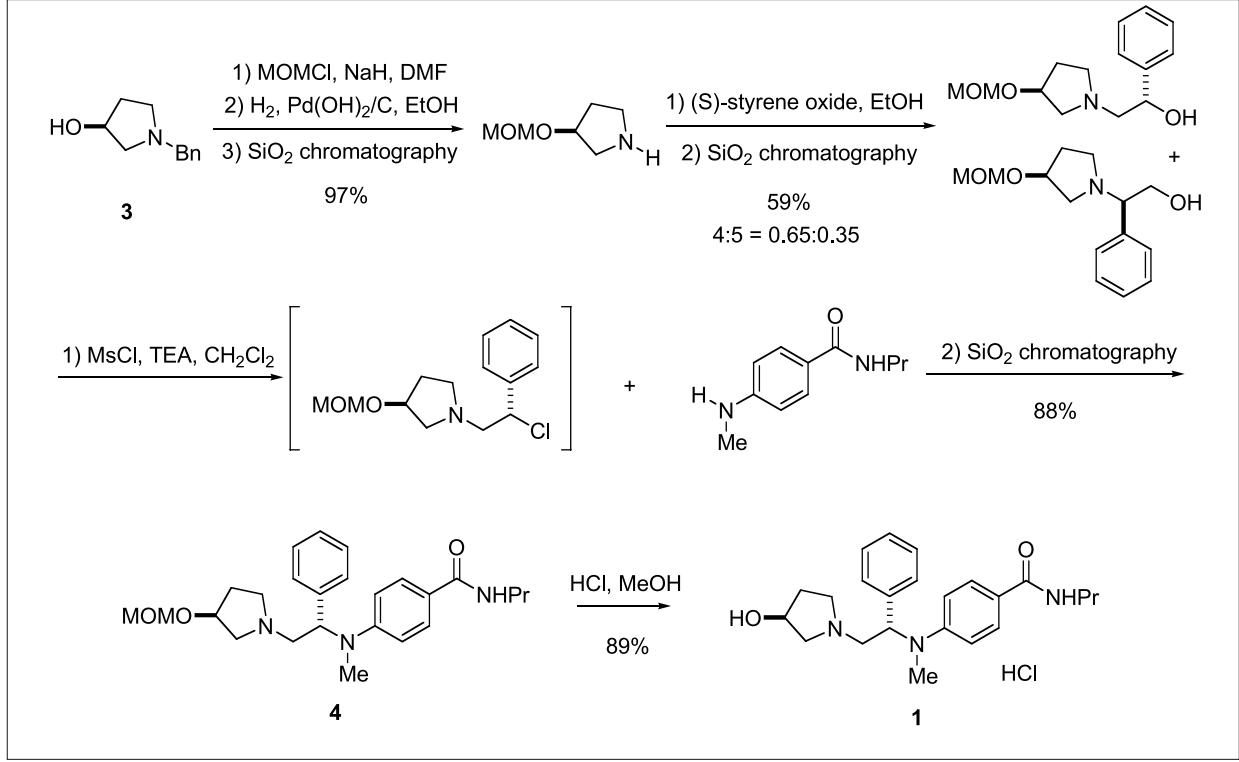

Scheme 1. Original discovery route



Scheme 2.



Scheme 3
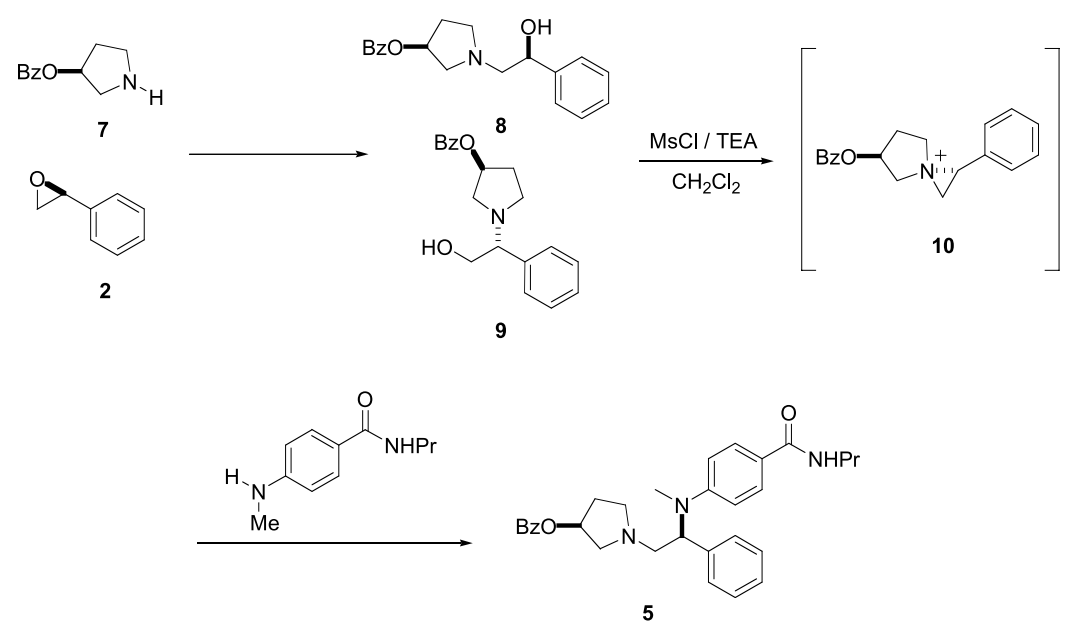

Scheme 4

timate stage would be too time consuming. Instead, we reasoned that sacrificing some of the scarce drug-substance to directly install the protecting group would provide the answer we were seeking in a single step instead of four.

Thus, using the bottom-up approach [3], we derivatized the alcohol group of $\mathbf{1}$ in various protected forms (Scheme 2) compatible with the previous chemistry, and quickly identified the benzoate ester $\mathbf{5}$ as a stable crystalline intermediate, easily isolable from ethyl acetate/hexane. On the basis of this approach N-benzyl pyrrodinol (3) was protected as the benzoate ester 6 (Scheme 3). By performing the reaction in the absence of base, the hydrogen chloride generated in the process provided the hydrochloride salt, which was conveniently isolated as a crystalline solid by addition of MTBE upon reaction completion. In addition to the hydrochloride's crystallinity, the subsequent N-benzyl hydrogenolysis with $10 \% \mathrm{Pd} / \mathrm{C}$ in isopropanol is accelerated as compared to the corresponding oily freebase, which actually stalls at some point. Upon completion of the reaction and filtration to remove the catalyst, the solvent is concentrated with concomitant azeotropic removal of water originating from the wet catalyst. The resulting solution was then diluted with diisopropyl ether, from which pyrrolidine 7 crystallized out of the solution. When performed on multi-kilo scale, the reactions yielded respectively 97 and $87 \%$ of easily filterable crystalline solids.

With the requisite pyrrolidine $\mathbf{7}$ in hand, the key coupling with $(S)$-styrene oxide was investigated. To that effect, the preparation of $\beta$-substituted amines from mixtures of styrene oxide opening products via a common aziridinium ion intermediate was recently communicated [4]. In essence, the production of the two regioisomers 8 and $\mathbf{9}$ is not problematic since they both independently lead to the desired diamine $\mathbf{5}$ via the intermediacy of aziridinium $\mathbf{1 0}$ (Scheme 4).

The regioisomeric mixture of $\mathbf{8}$ and $\mathbf{9}$ was an oil, whereas the isolated benzylic alcohol $\mathbf{8}$ is a stable, crystalline intermediate with a melting point of $103-104{ }^{\circ} \mathrm{C}$. Therefore, from a process chemistry perspective it would be desirable to identify a regioselective synthesis of $\mathbf{8}$, which could be purified by simple crystallization, and thus provide an additional point of purity control in the synthesis.

The preferred solvent for the free-basing procedure was 2-MeTHF, which provided good solubility for the free base, and excellent separation from water, as compared to THF. It was later observed that while residual amounts of up to $10 \%$ 2-MeTHF left behind after displacement in the reaction solvent slowed the reaction slightly, it did not adversely affect the isomer ratio or impurity profile. Solvent screening was performed to optimize the 65:35 regioselectivity (8:9) observed in the original coupling reaction when performed in ethanol. In general, higher boiling, more polar solvents both increased the reaction rate and the ratio of 8:9. Performing the coupling in $\mathrm{N}$-methylpyrrolidinone at $100{ }^{\circ} \mathrm{C}$ offered the best regioselectivity (12:1), with reaction completion after $12 \mathrm{~h}$.

In the course of our experiments, we found that combination of NMP with water as an anti-solvent selectively crystallized the predominant regioisomer $\mathbf{8}$. The optimized procedure for coupling was to prepare the free-base of pyrrolidinium 7 in 
2-MeTHF, displace into NMP, add styrene oxide, heat to $100{ }^{\circ} \mathrm{C}$ for $12 \mathrm{~h}$, cool, add water, and filter. This procedure was exemplified in the kilo-lab facility [5] on $7.2 \mathrm{~kg}$ scale, resulting in isolation of $5.9 \mathrm{~kg}(60 \%)$ of crystalline aminoalcohol $\mathbf{8}$.

As this compound was considered to be a potential regulatory starting material in the NDA filing it would be desirable to have several suppliers and several syntheses would facilitate cost reduction. So while the above chemistry was being developed, we sought an alternative synthesis of the crystalline aminoalcohol $\mathbf{8}$. It is noteworthy that long-term stability testing of 8 at $25{ }^{\circ} \mathrm{C}, 70 \%$ relative humidity showed no detectable formation of impurities. Thus, starting from the current aminobenzoate 7 , alkylation with bromoacetophenone was performed to access the requisite ketone 11, which we hoped to reduce diastereoselectively to the enantiomerically pure alcohol 8 (Scheme 5). Catalyst screening was undertaken and led to identification of highly diastereoselective reactions conditions using $\operatorname{Ir}(\mathrm{COD})_{2} \mathrm{BF}_{4}$ in combination with $(R)-(S)$-Josiphos ligand to provide the crystalline aminoalcohol 8 in $95 \%$ de.

With alcohol $\mathbf{8}$ in hand, we proceeded with the key aziridinium formation and coupling (Scheme 6). We found that methylene chloride was an ideal solvent for the mesylation/chloride displacement reaction, and that triethylamine, required for both the activation and coupling steps, could be added in one initial charge. We also found that dichloromethane could advantageously be used in the coupling step instead of ethanol. Exclusion of the latter offered a single solvent process, eliminating a reaction quench and solvent swap. Additionally, the coupling occurred at a lower temperature, and there was no longer the need to remove the ethanol prior to the aqueous quench. Furthermore any residual pyrrolidine 7 was purged with two $1 \mathrm{M}$ hydrochloric acid washes. Methylene chloride, with its low boiling point, made for easy displacement with ethyl acetate, and subsequent crystallization of product via hexane addition and seeding. The penultimate intermediate was isolated in $87 \%$ yield ( $>99 \%$ purity) as a crystalline free-base. The process was tested on $5.9 \mathrm{~kg}$ scale providing $7.2 \mathrm{~kg}$ of pure (99\%), crystalline material.

The final transformation leading to the drug substance entails a saponification, followed by salt formation using benzoic acid in the presence of water to produce the mono-hydrated salt. Since the conjugate acid of the sodium benzoate produced in the saponification is the actual acid used to produce the final salt form, there lies an opportunity to neutralize the saponification reaction mixture with benzoic acid, in

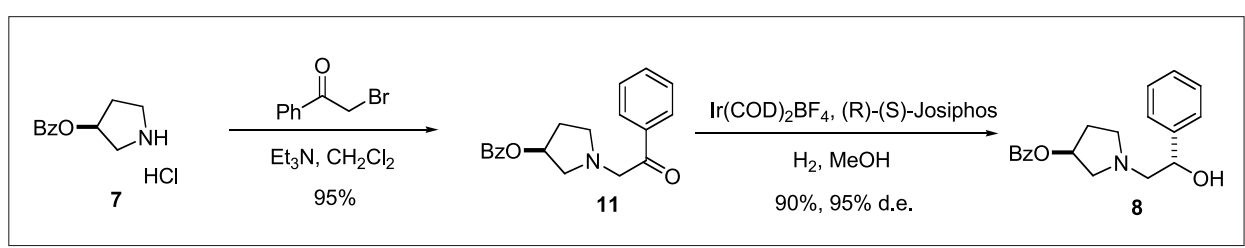

Scheme 5

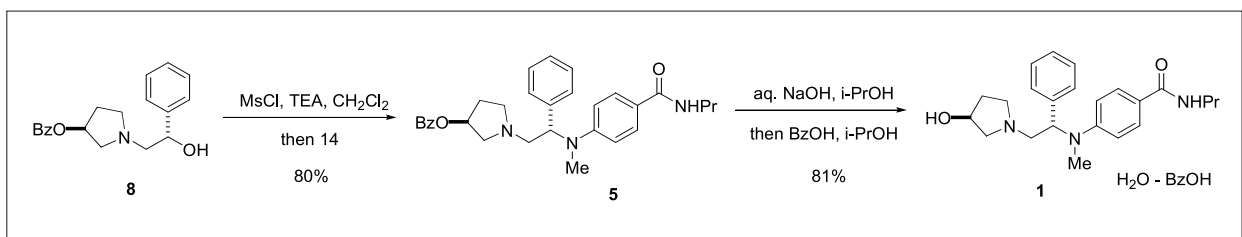

Scheme 6



Scheme 7 .

which case there cannot be scrambling between different counter-ions. Accordingly, instead of going through the usual series of $\mathrm{pH}$ adjustments and biphasic extractions, we devised a simple one-pot, one-isolation process. Following treatment of benzoate $\mathbf{5}$ with 1.2 equiv. of $1 \mathrm{M}$ sodium hydroxide for $4 \mathrm{~h}$ at $50{ }^{\circ} \mathrm{C}$ in 5 volumes ( 1 of solvent per $\mathrm{kg}$ of substrate) of isopropanol, 2.3 equiv. of benzoic acid were added to neutralize the excess sodium hydroxide and form the desired benzoate salt. The procedure was tailored such that when the clear solution is cooled to room temperature, the product crystallized out while the sodium benzoate by-product remained soluble. Following this simple process, the crystalline material was isolated by filtration to provide an $83 \%$ yield of drug substance $\mathbf{1}$, in the correct polymorphic state on multi-kilo scale.

\subsection{Process Research Towards Alternative Routes}

As mentioned earlier, a major limitation to the original synthetic route involving chiral oxirane $\mathbf{2}$, besides low overall yield and the non-crystalline nature of the intermediates, was the poor regioselectivity during epoxide ring-opening resulting in an inseparable mixture of regioisomers 8,9. Most of the problems were addressed during the aforementioned bulk campaign that made the process more efficient with a better control over the synthetic sequences and secured the supply for pharmacological studies. A rewarding outcome of this development work was the ability to obtain the crystalline penultimate intermediate $\mathbf{5}$. However, while improvement of the existing route was in progress for timely delivery of the initial bulk material, the search for new syntheses for long-term commercial production was initiated. The following sections describe two alternative approaches to $\mathbf{5}$ that resulted from our ongoing search for improvement, and explore an alternative disconnection relying on formation of the aniline residue by a $\mathrm{C}-\mathrm{N}$ bond forming step. Thus, for long-term commercial production of the API, we envisioned that a straightforward approach to these systems could rely on a transition metal-mediated amination as the key step involving derivatives of phenylglycine as the chiral amine source, and would also help eliminate some of those limitations of the early discovery route (Scheme 7).

\subsubsection{The Evolution of the}

\section{Pd-Catalyzed Amination Strategy [6]}

Significant progress has been made in the development of Pd-catalyzed crosscoupling of amines and aryl halides in recent years [7]. The industrial scope of these processes has further improved due to the availability of conditions to utilize relatively inexpensive aryl chlorides [8]. We became interested in this area in the course of developing a fundamentally different synthetic strategy to $\mathbf{1}$ that would present regulatory and synthetic advantages (e.g. convergent, amenable to scale up).

\subsubsection{1. $S_{N}$ Ar vs. Pd-Catalyzed Amination: A Model Study}

Preliminary experiments with N-methyl benzyl amine and 4-fluorobenzamide showed an extremely sluggish $\mathrm{S}_{\mathrm{N}} \mathrm{Ar}$ (at best $40 \%$ in situ yield in DMSO) even at elevated temperature. On the other hand, the corresponding aryl bromides showed promising results when palladium-mediated coupling 


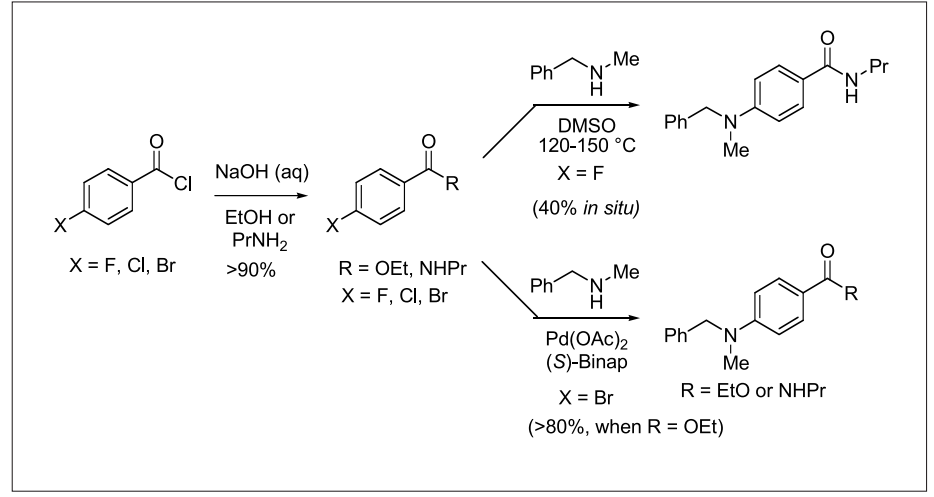

Scheme 8. Pd-catalyzed amination of the model

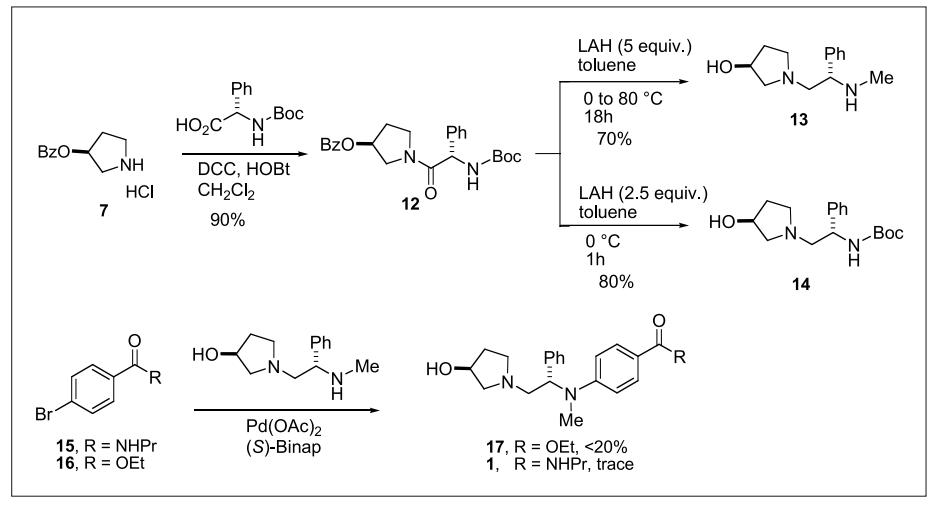

Scheme 9. Amide reduction approach to CJ-15,161 conditions were used with either the ester or the amide (Scheme 8).

\subsubsection{First Synthesis of 1 involving Amide Reduction and Pd-catalyzed Amination}

Encouraged by the results from the amination sequence, we prepared the desired secondary amine following standard procedures. 3-Pyrrolidinol benzoate (7) was coupled (DCC/HOBT) with Boc-protected Ph-Gly to obtain the corresponding amide 12 in high yield with no detectable epimerization (Scheme 9). Simultaneous reduction of the amide carbonyl, reductive removal of the benzoate, and reduction of the N-Boc of 12 to the N-Me derivative $\mathbf{1 3}$ was efficiently done in one pot in presence of excess LAH. Although exhaustive reduction of the carbamate required heating to about $80^{\circ} \mathrm{C}$, reduction of the amide and the benzoate were rapid at $0{ }^{\circ} \mathrm{C}$. Thus, a controlled reduction followed by quench at $0{ }^{\circ} \mathrm{C}$ could be used to isolate Boc-protected amine $\mathbf{1 4}$ in high yield, cleanly.

With the desired amine $\mathbf{1 3}$ in hand, we next attempted the aryl amination sequence. However, amination using amino alcohol 13 proved to be extremely sluggish under a variety of conditions. Using $\mathrm{Pd}(\mathrm{OAc})_{2} /(S)$ BINAP reagent system very little reaction was observed with amide $\mathbf{1 5}$, and less than $20 \%$ isolated yield with the ester 16, probably due to the very nature of the amino alcohol $\mathbf{1 4}$ as well as the amide-containing aryl bromide $\mathbf{1 5}$ [9]. Brief attempts to improve the yield by changing the Pd or phosphine source, reaction conditions, and with protection of the hydroxy group (TBDMS) did not show improvement to any appreciable extent. We were, however, pleased to find that the corresponding primary amine $\mathbf{1 9}$ as the TBDMS ether $\mathbf{2 0}$ did produce the coupled product $\mathbf{2 1}$ in a reasonable yield (Scheme 10). Amination using the combination of $\mathrm{Pd}(\mathrm{Oac})_{2}, t$-BuONa and an appropriate phosphine ligand [10] in toluene at $100{ }^{\circ} \mathrm{C}$ overnight followed by acidic (aqueous $\mathrm{HCl}$ at $50{ }^{\circ} \mathrm{C}$ ) deprotection provided the desired alcohol 22 in $80 \%$ yield. Meth- ylation of the aryl amine $\mathbf{2 2}$ under usual conditions produced the desired N-methyl derivative 1 in $~ 40 \%$ yield. It is, however, noteworthy to mention that the palladiummediated coupling was not successful even with the primary amine $\mathbf{1 9}$ when the alcohol was unprotected.

Although we were able to demonstrate the feasibility of the synthetic sequence described above for production of CJ-15,161 (1), several issues remained to be addressed before we could consider it for scale-up. One difficulty in using the primary amino alcohol $\mathbf{1 9}$ as substrate was its high hydrophilicity. When the BOC deprotection was performed with acid to generate the primary amine 19, recovery was low from the aqueous work-up even after proper $\mathrm{pH}$ adjustment. Thus, the need for a protecting group stable to the LAH reduction as well as BOC deprotection conditions was apparent. Such protecting groups would ideally serve to improve the hydrophobic properties of the molecule and ease the work up procedure.

\subsubsection{Exploring Alternatives to 'Amide Reduction' Approach}

While proper choice of protection/deprotection strategy remained a challenge, we evaluated a modified approach to our key amine fragment. To avoid scale-up related issues including epimerization of amide 12, we decide to use phenylglycinol 24 instead of the amino acid as the chiral amine source (Scheme 11). Preparation of the Boc-protected mesylate $\mathbf{2 5}$ and tosylate $\mathbf{2 6}$ were straightforward on the basis of precedent in the literature [11]. Protection of phenylglycinol with $\mathrm{Boc}_{2} \mathrm{O}$ followed by treatment with either mesyl or tosyl chloride proceeded in high yield, however, coupling of either $\mathbf{2 5}$ or $\mathbf{2 6}$ with pyrolidinol 27 was not efficient. The most common byproduct observed from these reactions was the oxazolidinone 30, presumably by intramolecular displacement of the intermediate thioester with the carbamate carbonyl [12].

Attempts to displace the mesylate of Boc-protected phenylglycinol with excess aminobenzoate $\left(45-50{ }^{\circ} \mathrm{C}\right.$; THF, toluene or $\mathrm{DMF}$ ) produced a mixture of starting materials $\mathbf{2 5}$ and $\mathbf{7}$, desired product $\mathbf{2 9}$, and oxazolidinone 30 [13], which were difficult to separate.

\subsubsection{4. 'Simultaneous Protection/ Activation' Strategy via Oxathiazolidine Formation}

In order to minimize byproduct (e.g. oxazolidinone 30) formation, and to provide an effective leaving group compatible

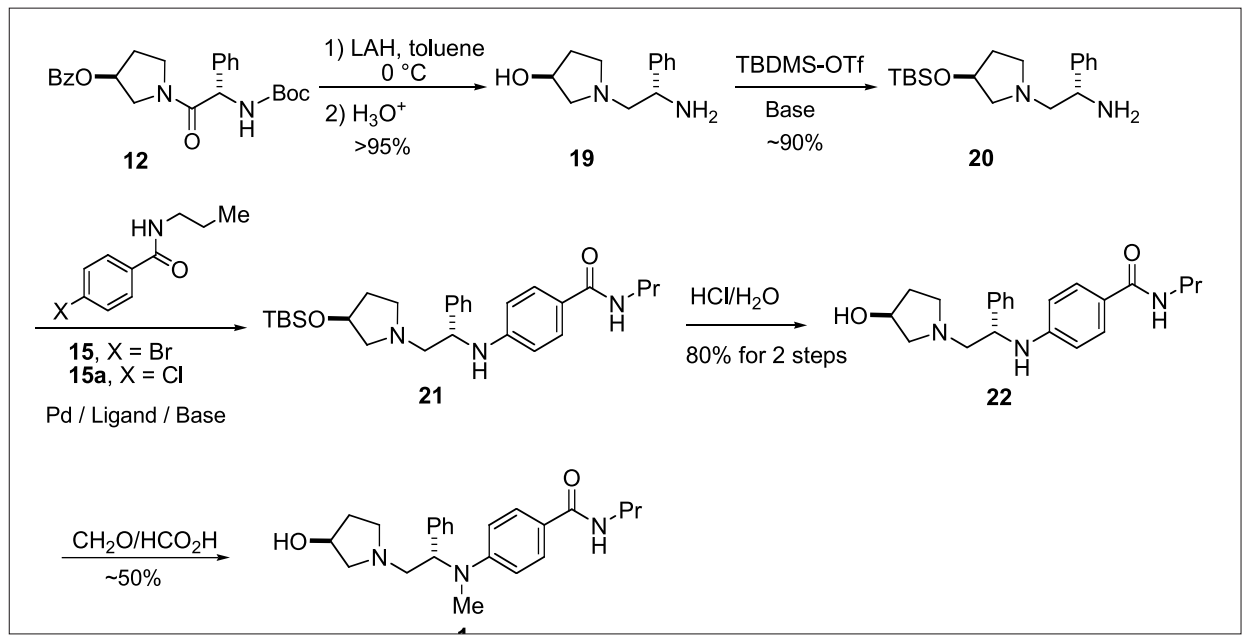

Scheme 10. First generation synthesis of CJ-15,161 (1) via C-N coupling 
with protection of the amine, an alternative strategy was followed (Scheme 12). To this end, treatment of Boc or Cbz protected phenyl glycinols $\mathbf{3 1}$ with $\mathrm{SOCl}_{2}$ in presence of $\mathrm{Et}_{3} \mathrm{~N}$ produced the cyclic 1,2,3-oxathiazolidine 2-oxide 32 which after oxidation provided 1,2,3-oxathiazolidine 2,2-dioxide $33(\sim 50-60 \%)$. Two major impurities were identified from the $\mathrm{SOCl}_{2}$ step during scaleup runs; the oxazolidinone $\mathbf{3 0}$ was generated once again and the dimer $\mathbf{3 4}$ was formed [14]. With appropriate choice of the base (pyridine) and reaction conditions (reverse addition), formation of these impurities was nearly eliminated and isolated yield of $\mathbf{3 2}$ increased significantly (98\%).

For the oxidation of the oxathiazolidine oxides $\mathbf{3 2}$ to the dioxides $\mathbf{3 3}$, a variety of oxidation conditions were screened to avoid decomposition due to over oxidation, partial deprotection (e.g. Boc), or hydrolytic cleavage. Among them, $\mathrm{NaIO}_{4} / \mathrm{RuCl}_{3}$ conditions proved superior providing a very clean product. Poor yield due to over oxidation was controlled by proper optimization of the reaction conditions, and the desired dioxides 33a,b were isolated in excellent yield with Boc-or Cbz-phenylglycinol $(\mathbf{2 2 a}, \mathbf{b})$ as the only detectable byproducts.

Nucleophilic displacement of the cyclic sulfamidates 33a,b with the benzoate protected pyrrolidinol [15] and removal of protecting groups was straightforward and high yielding. Both protected substrates $\mathbf{3 3 a}, \mathbf{b}$ coupled easily at room temperature with the aminobenzoate.

The sulfamic acids $\mathbf{3 4 a}, \mathbf{b}$ could be isolated in $60-70 \%$ recrystallized yield as the stable zwitterion, which after hydrolysis with acid gave the desired amines 35a,b quantitatively (Scheme 13) [16]. The Bocprotecting group was also removed under these conditions and provided the primary amine 35a directly. The $\mathrm{Cbz}$ group of 35b was removed to obtain 35a cleanly under transfer hydrogenation conditions $\left(\mathrm{HCO}_{2} \mathrm{NH}_{4}, \mathrm{Pd} / \mathrm{C}\right)$.

A variety of palladium/ligand combinations under a wide range of conditions were explored for the key amination sequence. The palladium-mediated coupling of the benzylamine 35a with the aryl bromide $\mathbf{1 5}$ using $\mathrm{Pd}(\mathrm{OAc})_{2} / \mathrm{BINAP}$ catalyst system provided a modest $\sim 39 \%$ yield [17]. Notably, $\mathrm{Pd}_{2}(\mathrm{dba})_{3} /(o$-biphen $) \mathrm{P}(t \text {-Bu })_{2}$ combination proved to be more efficient when the corresponding aryl chloride was used ( $c a$. $42 \%$ ). The separation of residual starting material proved to be very difficult. So an effort was made to identify a process that would cleanly produce the N-methylated product.

Reductive alkylation in presence of paraformaldehyde, using $\mathrm{NaBH}_{4} /$ Lewis acids or aqueous formaldehyde and STAB or $\mathrm{NaH}_{2} \mathrm{PO}_{3}$ combination showed some prod-

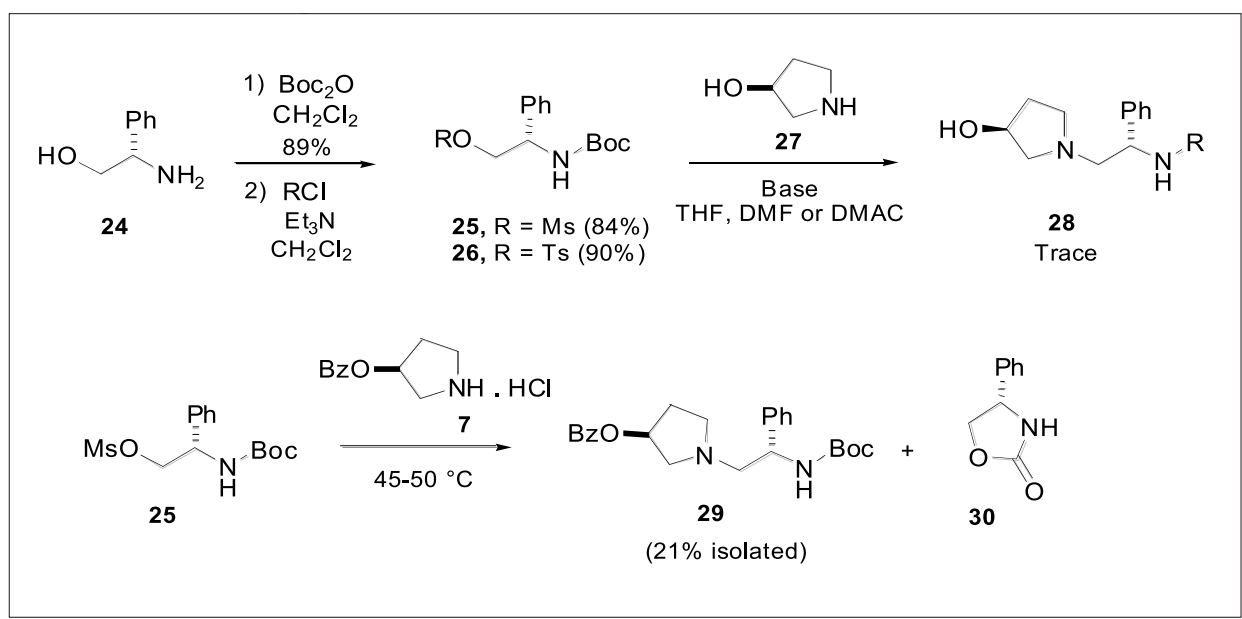

Scheme 11. Towards a preferred route to CJ-15,161
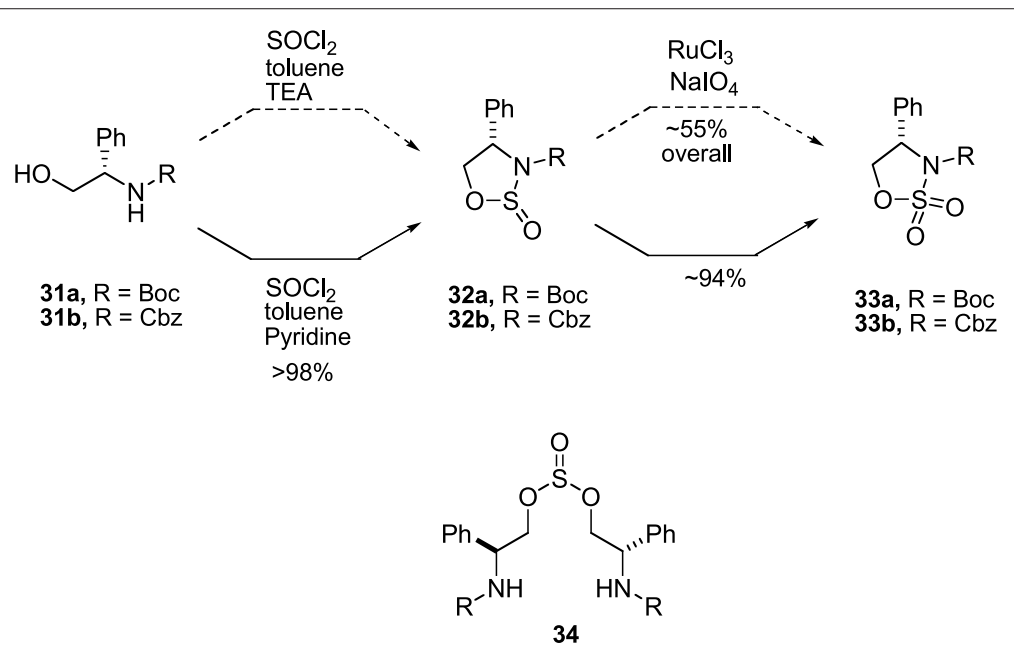

Scheme 12. Simultaneous protection/activation strategy via oxathiazolidine formation

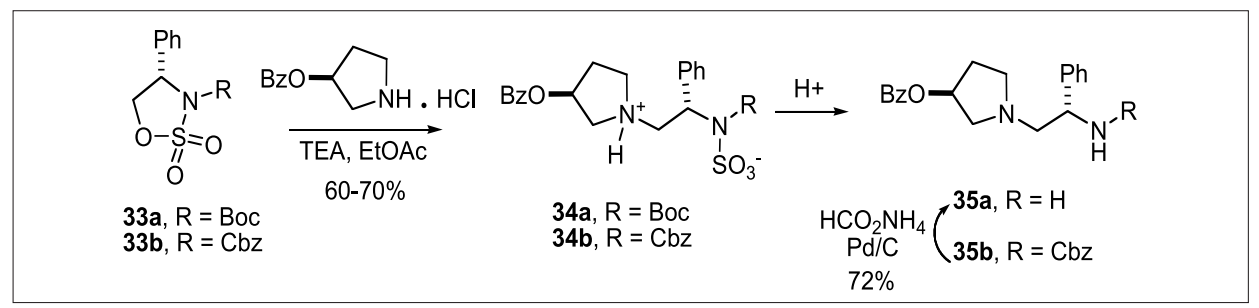

Scheme 13. Preparation of 1,2-diamine via $\beta$-aminosulfamic acids

uct but the reaction did not go to completion even after prolonged reaction time with excess reagent. However, a combination of $\mathrm{NaCNBH}_{3}, \mathrm{TMSCl}$, and $\mathrm{MgSO}_{4}$ proved to be the most promising conditions in producing the desired N-methylated product in good yield. Scheme 14 summarizes the successful completion of the synthetic sequence for the synthesis of penultimate intermediate $\mathbf{5}$.

Although more work still needs to be done to optimize and improve the efficiency of this process for practical application in an industrial sense, it constitutes a formal scalable synthesis of CJ-15,161 (1) involving Pd-catalyzed aryl amination as the key-step. The direct amination of an amide-containing aryl halide as well as efficient preparation of the substrate amine by dual protection/activation involving oxathiazolidinone formation are two notable features of the synthetic scheme.

While this work was in progress, we were also exploring other cross-coupling strategies that would give us easy access to the desired framework. As the Buchwald [18] and Hartwig [7b] groups have independently demonstrated, the generality of Pd-catalyzed $N$-arylation could be extended to amides and acyclic carbamates [19]. We reasoned that the byproduct oxazolidinone 30 could also potentially serve as the sub- 


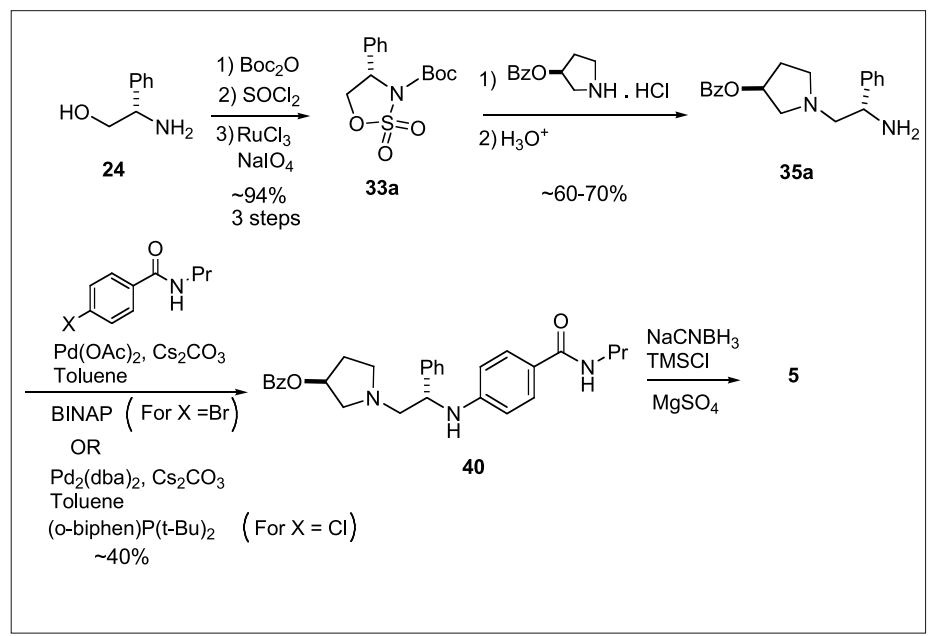

Scheme 14. Synthesis of penultimate intermediate $\mathbf{5}$ involving Pd-catalyzed aryl amination

strate for amidation. Thus, we explored $\mathrm{N}$-arylation of oxazolidinones [20], and reported a method involving a Pd-catalyzed C-N cross-coupling process between aryl chlorides and oxazolidinones which provided a convergent access to such systems in relatively few steps (Scheme 15). These derivatives could also be used as surrogates to produce a diverse array of enantiopure $\beta$-amino alcohols for further synthetic manipulation.

\subsubsection{Cu-catalyzed N-Arylation of} Oxazolidinones: An Efficient Approach to 1 [21]

Recently, Buchwald and coworkers have further improved the scope of traditional $\mathrm{Cu}$-catalyzed cross-couplings under Goldberg-type conditions [22]. Traditionally these involved stoichiometric copper reagents in a high boiling solvent at high temperature. These reactions can now be performed using catalytic copper $(<1-10$ $\mathrm{mol} \%$ ) in the presence of a bidentate amine ligand, and an industrially accessible base (e.g. $\mathrm{K}_{3} \mathrm{PO}_{4}$ or $\mathrm{K}_{2} \mathrm{CO}_{3}$ ). These conditions tolerate many functional groups that are known to be problematic in palladiumcatalyzed coupling reactions, and provide a cost-effective and practical method for $\mathrm{N}$ arylation. Thus, concurrent to developing conditions for Pd-catalyzed N-arylation of oxazolidinones, we explored $\mathrm{Cu}$-catalyzed conditions as a more viable commercial alternative. This concluding section describes a mild, practical, and scalable synthetic method for $\mathrm{N}$-arylation of oxazolidinones in general using relatively efficient (cost, operational) $\mathrm{Cu}$-catalyzed conditions.

The reaction of oxazolidinones with aryl halides in the presence of catalytic $\mathrm{Cu}$ was demonstrated to be fairly general over a variety of substrates. The coupled N-arylated oxazolidinones were obtained in high yield in most of the cases. Interestingly, the reaction turned out to be less sensitive to the electronic nature of the substituent of the substrate aryl halides (compared to Pd-catalyzed coupling conditions), and the oxidation state of the copper catalyst $(0,1$ or 2) proved not to be as crucial as is usually thought. The mechanism of this process is not well established. It is possible that the addition of a bidentate amine ligand not only helps solubilize copper salts, but also promotes the copper (I)/(II) disproportionation and stabilizes the active copper (I) species.

Thus, the key step in the synthesis was the coupling of the $p$-amido aryl bromide (15) with the oxazolidinone 21 (Scheme 16). Hydrolytic deprotection of the $\mathrm{N}$-aryl oxazolidinone 36 in ethanolic $\mathrm{NaOH}$ gave the aminoalcohol 37. Efficient conversion of $\mathbf{3 7}$ to 5 involved dual protection/activation involving oxathiazolidinone 39, nucleophilic ring opening of $\mathbf{3 9}$ to the 1,2-diamine $\mathbf{4 0}$ with pyrrolidinol benzoate ester derivative followed by reductive methylation.

\section{Concluding Remarks.}

The strategies adopted during process research and scale-up of the $\kappa$-opioid receptor agonist CJ-15,161 drug candidate are a typical example of process chemistry. The first part of this account demonstrates how rapid improvement of the existing protocol enabled the large-scale synthesis of CJ-15,161, where all the intermediates are crystalline solids, and only one biphasic extraction is utilized throughout the synthesis. The end-game of this route features a saponification quench with benzoic acid, which concomitantly produces the desired benzoate final salt form directly in the correct polymorphic state. An efficient four-step sequence was developed during this work, where four consecutive regioselective and stereospecific inversions at a single aziridinium stereogenic center lead

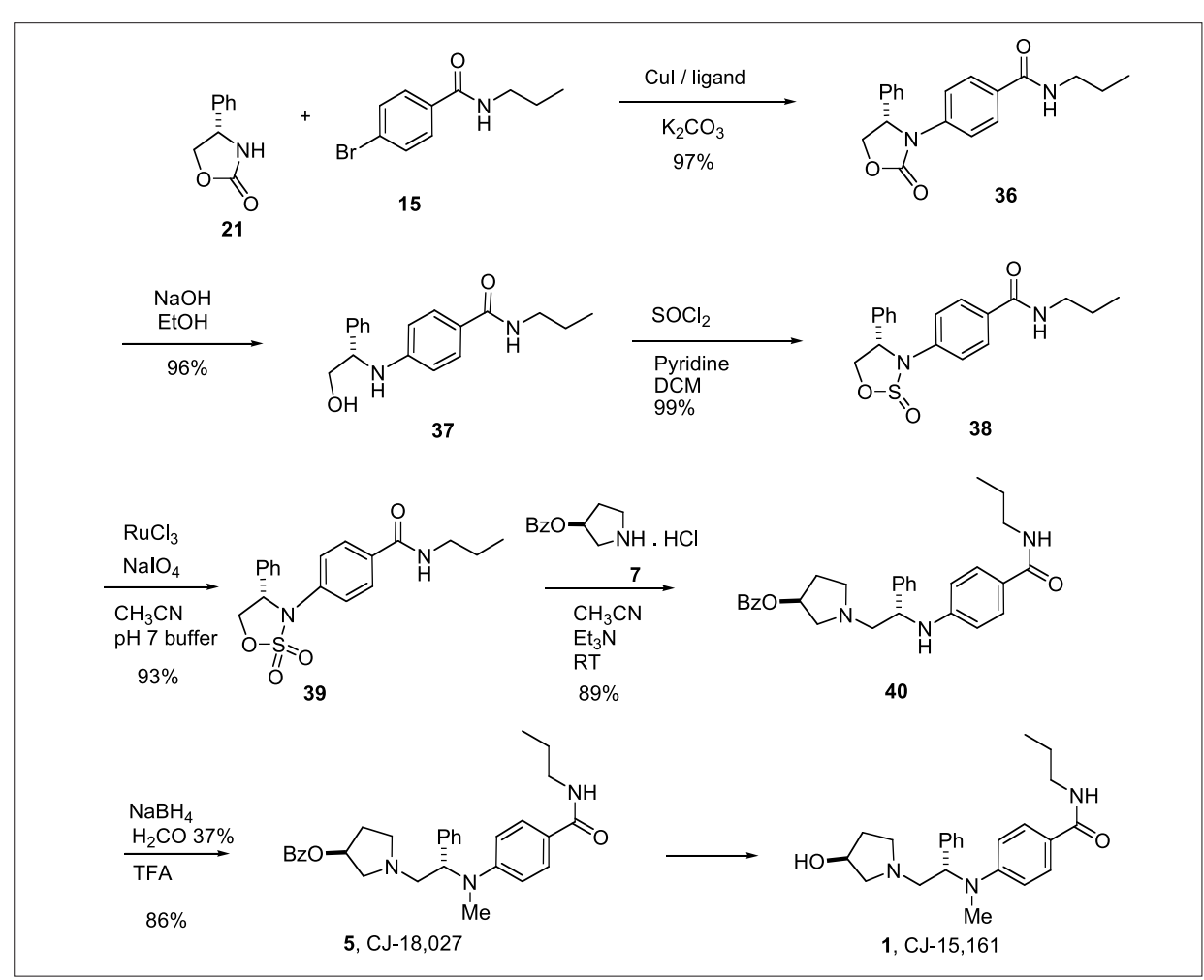

Scheme 16. Formal synthesis of $\mathbf{1}$ involving Cu-catalyzed aryl amination 
to overall retention of the stereochemistry, in a single operation. The concluding parts describe two convergent methods involving efficient intermolecular $\mathrm{N}$-arylation strategies. The first approach involves Pdcatalyzed cross-coupling between an aryl halide and an appropriately functionalized diamine, obtained from the precursor $\alpha$ amino acids or, more conveniently, from the corresponding 1,2-amino alcohols. The direct amination of 4-halo benzamide [22a] as the halide counterpart, and efficient preparation of the substrate amine by dual protection/activation involving oxathiazolidine formation are two noteworthy transformations of the synthetic scheme. The second approach exploits efficient intermolecular $\mathrm{N}$-arylation of oxazolidinones using catalytic copper in the presence of a bidentate ligand leading to a straightforward and practical synthesis of CJ-15,161.

In summary, this article illustrates a typical process evolution paradigm currently observed within the pharmaceutical industry. The time-driven early exercise involves safe and rapid execution of multikilogram campaigns to supply material for early studies, often using a less than perfect synthetic protocols that require utmost emergency readiness, while longterm commercial synthetic designs consider efficiency, robustness, safety, and greenness of the processes for long-term production.

\section{Acknowledgments}

We thank Mr. Richard Patterson, Tom Staigers, Thomas St. Louis and co-workers for their assistance in successfully transferring the chemical process to the pilot plant, and Mr. Kyle Leeman and Ms. Ioulia Loubkina for their analytical support. We sincerely thank Dr. Tamim Braish (Pfizer Inc.), Professors Steven Ley (Cambridge University), and David Collum (Cornell University) for helpful discussions.

\section{Received: June 17, 2006}

[1] F. Ito, H. Kondo, US Patent No. 6201007 B1, 2001.

[2] M. Couturier, J.L. Tucker, B.M. Andresen, K.M. DeVries, B.C. Vanderplas, F. Ito, Tetrahedron: Asym. 2003, 14, 3517-3523.

[3] C.-K. Chen, A.K. Singh, Org. Proc. Res. Dev. 2001, 5, 508.

[4] S.R. Anderson, J.T. Ayers, K.M. DeVries, F. Ito, D. Mendenhall, B.C. Vanderplas, Tetrahedron Asym. 1999, 10, 2655.

[5] E. Kobelski, T. Staigers, C. Sullivan, Pharm. Eng. 2002, 22, 1.

[6] A. Ghosh, J.E. Sieser, S. Caron, T.J.N. Watson, Chem. Comm. 2002, 15, 16441645.

[7] a) J.F. Hartwig, Angew. Chem., Int. Ed. 1998, 37, 2046-2067; b) J.F. Hartwig, M. Kawatsura, S.I. Hauck, K.H. Shaughnessy, L.M. Alcazar-Roman, J. Org. Chem.
1999, 64, 5575-5580; c) B.H. Yang, S.L. Buchwald, J. Organomet. Chem. 1999, 576, 125-146; d) J.P. Wolfe, S.L. Buchwald, J. Org. Chem. 2000, 65, 1144-1157; e) J.P. Wolfe, H. Tomori, J.P. Sadighi, J. Yin, S.L. Buchwald, J. Org. Chem. 2000, 65, 1158-1174 and references therein.

[8] For examples of chloroarene activation, see: a) V.V. Grushin, H. Alper, Chem. Rev. 1994, 94, 1047-1062; b) C. Dai, G.C. Fu J. Am. Chem. Soc. 2001, 123, 2719-2724.

[9] For some isolated examples of amide as substrate, see: a) Y.D. Ward, V. Farina Tetrahedron Lett. 1996, 37, 6993; b) C.A. Willoughby, K.T. Chapman, Tetrahedron Lett. 1996, 37, 7181; c) A. Batch, R.H Dodd, J. Org. Chem. 1998, 63, 872; d) J.T. Link, B. Sorensen, G. Liu, Z. Pei, E.B. Reilly, S. Leitza, G. Okasinski, Bioorg. Med. Chem. Lett. 2001, 11, 973.

[10] Generally, BINAP was used for $\mathrm{ArBr}$ and (o-biphen) $\mathrm{P}(t-\mathrm{Bu})_{2}$ was used for other $\mathrm{ArCl}$ substrates.

[11] G.A.M. Giardina, L.F. Raveglia, M. Grugni, H.M. Sarau, C. Farina, A.D. Medhurst, D. Graziani, D.B. Schmidt, R. Rigolio, M. Luttmann, S. Cavagnera, J.J. Foley, V. Vecchietti, D.W. P. Hay, J. Med. Chem. 1999, 42, 1053-1065.

[12] A similar mechanism has been proposed for the formation of 2-oxazolidone from $\beta$-iodourethane, see; C. Heathcock, A. Hassner, Angew. Chem. 1963, 75, 344.

[13] J.W. Lee, J.H. Lee, H.J. Son, Y.K. Choi, G.J. Yu, M.H. Park, Synth. Commun. 1996 , 83, 83-88. For a recent mechanistic study of this transformation, see; C. Agami, F. Couty, L. Hamon, O. Venier, Tetrahedron Lett. 1993, 4509-4512.

[14] For a report on the preparation of sulfamidate using sulfuryl chloride, see; D. Alker, K. J. Doyle, L.M. Harwood, A. McGregor, Tetrahedron Asymmetry 1990, $1,877-880$.

[15] For some accounts of nucleophilic ring opening of cyclic sulfamidates with different nucleophiles, see; a) L. Wei, W.-D. Lubell, Org. Lett. 2000, 2, 2595-2598; b) M. Atfani, L. Wei, W.D. Lubell, Org Lett. 2001, 3, 2965-2968; c) M.K. Pound, D.L. Davies, M. Pilkington, M.M. de Pina Vaz Sousa, J. D. Wallis, Tetrahedron Lett. 2002, 43, 1915-1918.

[16] An A2 mechanism has been proposed for the hydrolysis of sulfamic acid in aqueous acid, see; G.A. Benson, W.J. Spillane, Chem. Rev. 1980, 80, 151. For some examples of acid-hydrolysis of sulfamic acids, see; M. Okuda, K. Tomioka, Tetrahedron Lett. 1994, 35, 4585-4586; M.E. Van Dort, Y.W. Jung, P.S. Sherman, M.R. Kilbourn, D.M. Wieland, J. Med. Chem. 1995, 38, 810-815; B.M. Kim, S.M. So, Tetrahedron Lett. 1998, 39, 5381-5384.

[17] The yields are lower with benzoate protected substrate $\mathbf{3 5 a}$ due to competing hydrolysis of the starting amine. With silyl protection the $\%$ yields were in the $80 \mathrm{~s}$.
[18] a) J.P. Wolfe, R.A. Rennels, S.L. Buchwald, Tetrahedron 1996, 21, 7525-7546; (b) B.H. Yang, S.L. Buchwald, Org. Lett. 1999, 1, 35-37; c) J. Yin, S.L. Buchwald, Org. Lett. 2000, 2, 1101-1104; d) J. Yin, S.L. Buchwald, J. Am. Chem. Soc. 2002 , 124, 6043-6048

[19] a) For a related process, see: Z. Wang, R.T. Skerlj, G.J. Bridger, Tetrahedron Lett. 1999, 40, 3543-3546; b) J.B. Arterburn, K.V. Rao, R. Ramdas, B.R. Dible, Org. Lett. 2001, 3, 1351-1354; d) W.C. Shakespeare, Tetrahedron Lett. 1999, 40, 2035-2038; d) for intramolecular amidation, see: J.P. Wolfe, R.A. Rennels, S.L. Buchwald, Tetrahedron 1996, 52, 7525 7546.

[20] For a review on therapeutic oxazolidinones, see: a) E. Halle, J. Majcher-Peszynska, B. Drewelow, Chemotherapie J. 2002, 11, 1-11. For some related work in this area, see: b) J.A. Tucker, D.A. Allwine, K.C. Grega, M.R. Barbachyn, J.L. Klock, J.L. Adamski, S.J. Brickener, D.K. Hutchinson, C.W. Ford, G.E. Zurenko, R.A. Conradi, P.S. Burton, R.M. Jensen, J. Med. Chem. 1998, 41, 3727-3735; c) M.J. Genin, D.K. Hutchinson, D.A. Allwine, J.B. Hester, D.E. Hemmert, S.A. Garmon, C.W. Ford, G.E. Zurenko, J.C Hamel, R.D. Schaadt, D. Stapert, B.H. Yagi, J.M. Friis, E.M. Shobe, W.J. Adams, J. Med. Chem. 1998, 41, 5144-5147; d) D.M. Gleave, S.J. Brickner, P.R. Manninen, D.A. Allwine, K.D. Lovasz, D.C. Rohrer, J.A. Tucker, G.E. Zurenko, C.W Ford, Bioorg. Med. Chem. Lett. 1998, 8, 1231-1236; e) D.M. Gleave, S.J. Brickner, J. Org. Chem. 1996, 61, 6470-6474.

[21] A. Ghosh, J.E. Sieser, S. Caron, M. Couturier, K. Dupont-Gaudet, M. Girardin, $J$. Org. Chem. 2006, 71, 1258-1261.

[22] Improvements including use of catalytic copper salt as well as significantly milder conditions have been described: a) A. Klapars, J.C. Antilla, X. Huang, S.L. Buchwald, J. Am. Chem. Soc. 2001, 123, 7727-7729. The effect of various diamine ligands on the efficiency of the aryl amidation reaction has been reported: b) A Klapars, X. Huang, S.L. Buchwald, J. Am. Chem. Soc. 2002, 124, 7421-7428. For the first report on intramolecular amidations under relatively mild conditions, see: c) T. Ohsawa, T. Kametani, M. Ihara, Heterocycles 1980, 14, 277-280. For reviews on $\mathrm{Cu}$-catalyzed crosscoupling reactions, see: d) J. Lindley, Tetrahedron 1984, 40, 1435-1456; e) S.V. Ley, A.W. Thomas, Angew. Chem., Int. Ed. 2003, 42, 5400. 